\title{
A fresh look at an antiviral helicase
}

\author{
Leonid Gitlin ${ }^{1}$, Marco Colonna ${ }^{1}$ \\ ${ }^{1}$ Department of Pathology and Immunology, Washington University School of Medicine, Campus Box 8118, 660 South Euclid Avenue, \\ St.Louis, MO 63110,USA.mcolonna@pathology.wustl.edu
}

Cell Research (2007) 17:898-900. doi: 10.1038/cr.2007.95; published online 15 November 2007

In order to survive, all organisms must guard against viral infections. Recognition of viruses is accomplished via multiple sensors. Many mammalian proteins can recognize viral products, such as double-stranded RNA (dsRNA), yet few of them are known to induce interferon, the central antiviral messenger. Since interferon is indispensable for successful antiviral defense [1], the interferon-inducing sensors have been of particular interest. However, a clear understanding of such sensors has been elusive, and the first well-established sensor family, the toll-like receptors (TLRs), was described relatively recently [2]. Antiviral TLRs are positioned in the endosomes, where they report the appearance of viral genetic material (DNA, single- and double-stranded RNA). However, the question of potential virus sensors in the cytoplasm was left open. Given the particular effectiveness of intracellular dsRNA at inducing interferon, it was suspected that dsRNA-binding sensor molecules would be found in the cytoplasm.

The breakthrough came with the work of Fujita and coworkers [3]. In a screen for dsRNA sensors, they discovered RIG-I, which can induce interferon gene transcription. Interestingly, RIG-I mRNA is, in turn, itself induced by interferon. RIG-I consists of two principal parts: a Cterminal helicase domain, which binds dsRNA, and two $\mathrm{N}$-terminal CARD (caspase recruitment) domains arranged in tandem. RIG-I was soon found to be part of an RNA helicase family, whose other member, mda5, turned out to have the same domain structure. Apparently, mda5 also has a very similar function, as it is also capable of inducing interferon gene transcription in response to dsRNA [4]. However, RIG-I has an additional capability of responding to the 5'-triphosphorylated RNA $[5,6]$.

It is believed that binding of dsRNA to the helicase domain leads to changes in the conformation of the protein so that the CARDs are free to initiate a signaling cascade. Both RIG-I and mda5 use the same signaling adaptor, IPS1 (also called MAVS, Cardif, and VISA). Importantly, a version of RIG-I containing the CARDs but lacking the helicase domain, designated here as RIG-I ${ }^{\text {CARD }}$, is constitutively active. Indeed, the cDNA recovered in the original screen was truncated at the $5^{\prime}$ of the helicase domain [3].

To examine the importance of the putative interferoninducing helicases, RIG-I, mda5, and IPS-1 knockouts were made [7-10]. These mice have confirmed the importance of the helicases in antiviral response; notably, it appears that mda5 and/or RIG-I can respond to almost any RNA virus, with mda5 specializing on picornaviruses as well as some other positive-stranded RNA viruses, and RIG-I detecting negative-stranded viruses as well as flaviviruses. Surprisingly, though, while IPS-1 and mda5-deficient mice were viable and fertile, RIG-I knockouts demonstrated very poor viability, with only a few pups surviving several weeks after birth. The cause of lethality was liver degeneration at embryonic day 12.5-14.

There may be several explanations for this phenotype. First, it is possible that RIG-I is required for some other process, not related to virus recognition and signaling through IPS-1. Another possibility is that the structure of the targeted allele results in a neomorphic function in the liver. One potential mechanism for such neomorphic function is the fact that the targeted allele has the helicase domain of the protein disrupted, while leaving the promoter and the CARD domains intact. Therefore, the mutant allele, if expressed, may resemble a truncated version of RIG-I, similar to the constitutively active RIG-I described above. Kato et al. carefully examined RIG-I expression in the knockout and did not detect any RIG-I-related mRNA or protein; however, it remains possible that only certain cell types would carry such message. If so, the CARDs of RIG-I could indeed prove detrimental, as interferon and interferon-stimulated genes would be upregulated, potentially leading to further increase in the RIG-I ${ }^{\mathrm{CARD}}$ levels. One argument against such a scenario is the fact that heterozygous mice are born at the expected frequency and 
don't seem to exhibit any pathology; however, RIG-I ${ }^{\mathrm{wt}}$ may exert a moderating influence on RIG-I ${ }^{\text {CARD }}$ activity, which would be lost in the homozygous RIG-I ${ }^{\text {CARD }}$ context.

To address the question of RIG-I requirement for viability, it would be important to make a separate RIG-I knockout allele. This has just been accomplished by Zhu-Gang Wang and his colleagues. In a recent issue of Cell Research, they describe a new RIG-I deficient mouse strain, which is viable and fertile [11]. The new knockout allele is structured such that one half of the second CARD domain is removed together with a crucial helicase motif.

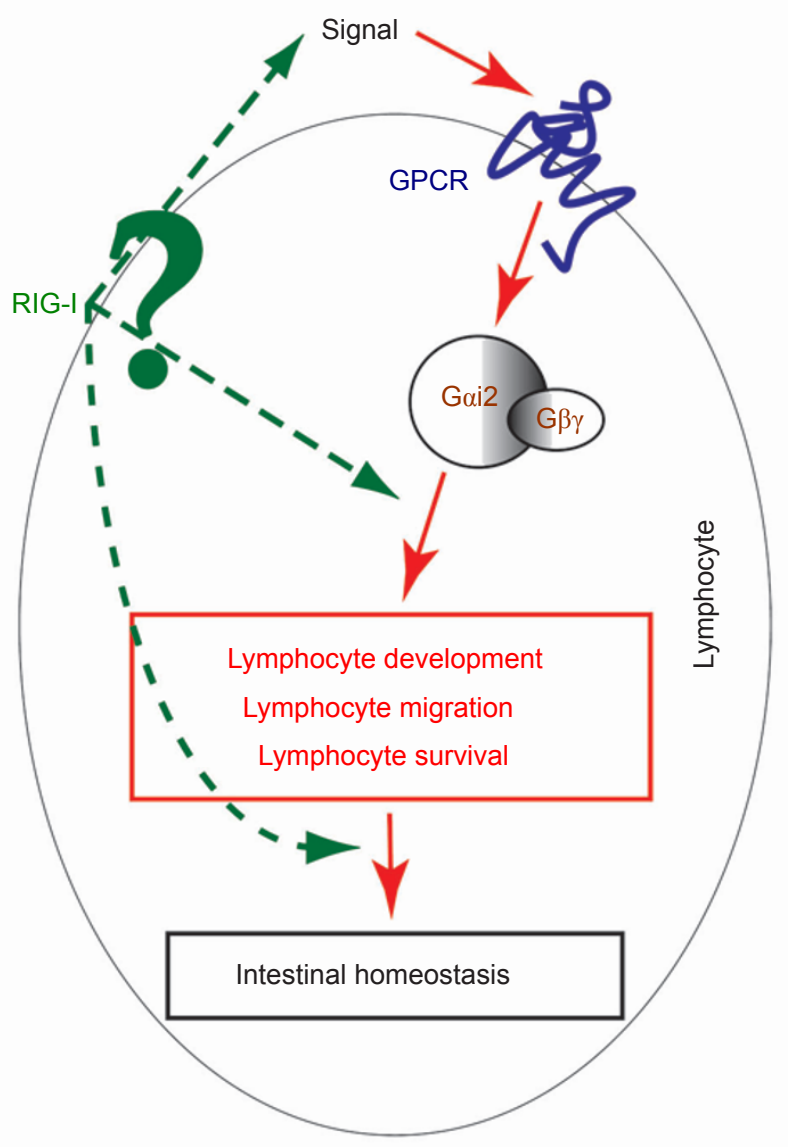

Figure 1 Gai2-dependent pathway of the intestinal immune homeostasis. In a recent issue of Cell Research [11], Wang and co-authors provide evidence that RIG-I may play a role at one or more points in the pathway. RIG-I knockout develops a colitis-like condition, similar to the knockout of Gai2. RIG-I ${ }^{-/}$lymphocyte populations are abnormal, suggesting that these cells, which are generally important in autoimmunity, are likely to be the site of primary RIG-I deficiency. However, the different abnormalities in the cell and tissues do not yet allow us to draw firm conclusions about the primary site or exact nature of the deficit. GPCR, G-protein coupled receptor; Gai2 and G $\beta \gamma$, G-protein subunits $\alpha \mathrm{i} 2$ and $\beta \gamma$.
Consequently, this design is not likely to lead to strong neomorphic functions. Additionally, the authors did not detect any RIG-I related mRNAs or protein.

While we do not know exactly what causes the difference in viability between the two knockout alleles, the availability of a viable RIG-I-deficient mouse strain opens the door to many new and interesting experiments, and the authors already report some intriguing findings. Specifically, the new strain is prone to developing a colitis-like condition. Interestingly, a point mutation in mda5 has been tied to a human autoimmune disease, type I diabetes [12]. However, the report of Wang and co-workers is the first to link RIG-I to autoimmunity, as well. The exact meaning of this finding is still unclear, and hypotheses explaining this phenomenon may be split into two broad categories. First, the absence of RIG-I may lead to an increased replication of a (presumably) viral pathogen, in turn triggering an aberrant immune response. Alternatively, RIG-I may be directly involved in the regulation of the immune homeostasis, such as a transcriptional response influencing the levels of certain mRNAs. Intriguingly, a deficiency in Gai2, which is an alpha subunit of a G-protein coupled receptor, leads to a colitis phenotype resembling that of the RIG-I knockout [13], as Peyer's patches are reduced in number and size, and peripheral T-cell subsets are skewed toward effector and memory cells. Indeed, the authors find that Gai2 promoter activity and mRNA levels are proportional to the RIG-I expression; however, more research will be required to definitively establish a causative, instead of a correlative, relationship between the two genes. If RIG-I acts in the same pathway, several scenarios are possible, as described in Figure 1.

And what of the virus susceptibility of the RIG-I deficient mice? After all, RIG-I appears to be a critical RNA virus sensor, based in large part on what we learned in vitro, in cells derived from the previous knockout. These experiments can now be performed in vivo, as well. Additionally, it is not certain that the outcome in the two different knockouts will be identical even under the in vitro conditions. We may be in for some unexpected discoveries, as the novel RIG-I knockout reveals new secrets of this RNA helicase.

\section{References}

1 Muller U, Steinhoff U, Reis LF, et al. Functional role of type I and type II interferons in antiviral defense. Science 1994; 264:1918-1921.

2 Takeda K, Akira S. Toll-like receptors in innate immunity.Int Immunol 2005; 17:1-14.

3 Yoneyama M, Kikuchi M, Natsukawa T, et al. The RNA helicase RIG-I has an essential function in double-stranded RNA-induced innate antiviral responses.[see comment]. Nat Immunol 2004; 
5:730-737.

4 Andrejeva J, Childs KS, Young DF, et al. The V proteins of paramyxoviruses bind the IFN-inducible RNA helicase, mda-5, and inhibit its activation of the IFN-beta promoter. Proc Natl Acad Sci USA 2004; 101:17264-17269.

5 Hornung V, Ellegast J, Kim S, et al. 5'-Triphosphate RNA Is the Ligand for RIG-I. Science 2006; 314:994-997.

6 Pichlmair A, Schulz O, Tan CP, et al. RIG-I-Mediated Antiviral Responses to Single-Stranded RNA Bearing 5'-Phosphates. Science 2006; 314:997-1001.

7 Kato H, Sato S, Yoneyama M, et al. Cell type-specific involvement of RIG-I in antiviral response. Immunity 2005; 23:19-28.

8 Kato H, Takeuchi O, Sato S, et al. Differential roles of MDA5 and RIG-I helicases in the recognition of RNA viruses. Nature 2006; 441:101-105.

9 Gitlin L, Barchet W, Gilfillan S, et al. Essential role of mda-5 in type I IFN responses to polyriboinosinic:polyribocytidylic acid and encephalomyocarditis picornavirus. Proc Natl Acad Sci USA 2006; 103:8459-8464.

10 Sun Q, Sun L, Liu HH, et al. The specific and essential role of MAVS in antiviral innate immune responses. [see comment]. Immunity 2006; 24:633-642.

11 Wang Y, Zhang HX, Sun YP, et al. Rig- $^{-{ }^{-/}}$mice develop colitis associated with downregulation of Galphai2. Cell Res 2007; 17:858-868.

12 Smyth DJ, Cooper JD, Bailey R, et al. A genome-wide association study of nonsynonymous SNPs identifies a type 1 diabetes locus in the interferon-induced helicase (IFIH1) region. Nat Genet 2006; 38:617-619.

13 Rudolph U, Finegold MJ, Rich SS, et al. Ulcerative colitis and adenocarcinoma of the colon in Galphai2-deficient mice. Nat Genet 1995;10:143-50. 